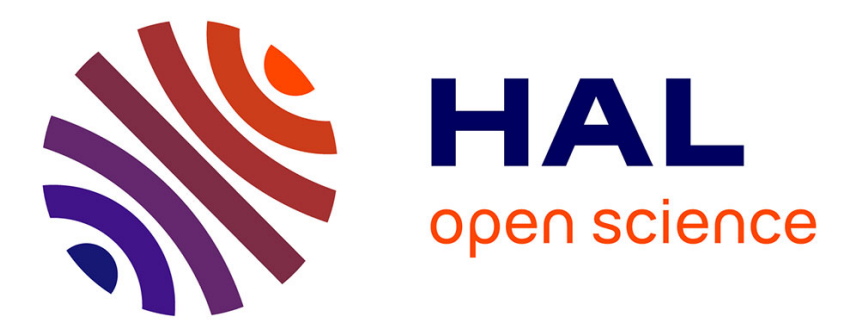

\title{
Effets de la température sur le taux de gonadotropine plasmatique et la spermatogenèse du poisson rouge Carassius auratus
}

\author{
Christian Gillet, Roland Billard, Bernard Breton
}

\section{To cite this version:}

Christian Gillet, Roland Billard, Bernard Breton. Effets de la température sur le taux de gonadotropine plasmatique et la spermatogenèse du poisson rouge Carassius auratus. Canadian Journal of Zoology, 1977, 55 (1), pp.242-245. 10.1139/z77-028 . hal-01604266

\section{HAL Id: hal-01604266 \\ https://hal.science/hal-01604266}

Submitted on 2 Jun 2020

HAL is a multi-disciplinary open access archive for the deposit and dissemination of scientific research documents, whether they are published or not. The documents may come from teaching and research institutions in France or abroad, or from public or private research centers.
L'archive ouverte pluridisciplinaire HAL, est destinée au dépôt et à la diffusion de documents scientifiques de niveau recherche, publiés ou non, émanant des établissements d'enseignement et de recherche français ou étrangers, des laboratoires publics ou privés.

\section{다(1)(2)}

Distributed under a Creative Commons Attribution - ShareAlikel 4.0 International 


\title{
Effets de la température sur le taux de gonadotropine plasmatique et la spermatogenèse du poisson rouge Carassius auratus
}

\author{
Christian Gillet, Roland Billard et Bernard Breton \\ Institut National de la Recherche Agronomique, Laboratoire de Physiologie des Poissons, 78350 Jouy-en-Josas, France \\ Reçu le 8 juin 1976
}

Gillet, C., R. Billard et B. Breton. 1977. Effets de la température sur le taux de gonadotropine plasmatique et la spermatogenèse du poisson rouge Carassius auratus. Can. J. Zool. 55: $242-245$.

Les teneurs en gonadotropine (c-GtH) plasmatique ont été mesurées sur des poissons rouges élevés à des températures constantes ou croissantes comprises entre 10 et $30^{\circ} \mathrm{C}$ de février à juillet. Aux températures d’élevage égales ou supérieures à $17^{\circ} \mathrm{C}$, les niveaux de c-GtH plasmatiques restent pendant 2 mois (avril et mai) significativement $(P<0.005)$ plus élevés qu'à $10^{\circ} \mathrm{C}$. En avril, la charge hypophysaire en c-GtH est maximum aux températures supérieures à $17^{\circ} \mathrm{C}$, et minimum au-dessous, alors qu'en juin, la situation est inverse. L'étude des gonades montre que la spermatogenèse est accélérée à 17 et $24^{\circ} \mathrm{C}$ par rapport à $10^{\circ}$, mais à $30^{\circ} \mathrm{C}$ l'activité spermatogénétique est inhibée.

Gillet, C., R. Billard, and B. Breton. 1977. Effets de la température sur le taux de gonadotropine plasmatique et la spermatogenèse du poisson rouge Carassius auratus. Can. J. Zool. 55: $242-245$.

Concentrations of immunoreactive plasma gonadotropin $(\mathrm{c}-\mathrm{GtH})$ were measured for goldfish kept at conditions of temperature held constant or increasing between 10 and $30^{\circ} \mathrm{C}$, between February and July. At temperatures of $17^{\circ} \mathrm{C}$ or above the levels of plasma c-GtH were significantly $(P<0.005)$ higher during April and May than at $10^{\circ} \mathrm{C}$. In April the pituitary concentration of $\mathrm{C}-\mathrm{GtH}$ was maximum for fish held at $17^{\circ} \mathrm{C}$ or above and the minimum for fish held below $17^{\circ} \mathrm{C}$, but in June the situation was reversed. A study of the gonads showed that spermatogenesis is faster at $17-24^{\circ} \mathrm{C}$ than at $10^{\circ} \mathrm{C}$, but at $30^{\circ} \mathrm{C}$ spermatogenic activity was inhibited.

La reproduction des poissons est fortement dépendante de l'environnement thermique qui peut conditionner à la fois le cycle reproducteur saisonnier (De Vlaming 1974) et la durée et l'efficacité de la gamétogenèse (Billard 1968). L'importance des effets de la température varie selon les espèces et on admet que le déterminisme du cycle gamétogénétique dépend de la température chez les Cyprinidés alors que la photopériode est le facteur le plus important chez les Salmonidés où la température n'a qu'un rôle facilitant (Breton et al. 1976). Les effets de la température sur la gamétogenèse restent mal connus chez les Cyprinidés et on ne dispose pas d'informations précises sur les niveaux thermiques compatibles avec une gamétogenèse normale. Dans la présente expérimentation, les effets de la température ont été testés sur la reproduction d'un cyprinidé, le poisson rouge, en prenant pour critères les niveaux gonadotropes circulants et le développement des testicules.

\section{Matériels et Méthodes}

Les poissons rouges agés de 3 à 4 ans et d'un poids moyen de $50 \mathrm{~g}$ ont été prélevés dans leurs bassins d'éle- vage placés à l'extérieur et mis en expérience (début de la période d'adaptation) le 10 janvier (température de l'eau, $10^{\circ} \mathrm{C}$ ). Le dispositif expérimental comporte des bassins de 601 thermorégulés $\left( \pm 1^{\circ} \mathrm{C}\right)$ dans chacun desquels se trouve un groupe de 10 animaux. Le protocole expérimental comporte cinq groupes qui sont soumis à des températures constantes $\left(10,17,24\right.$ et $30^{\circ} \mathrm{C}$; vitesse d'adaptation, $1{ }^{\circ} \mathrm{C}$ par jour) et deux groupes constituent les témoins (cf. bas de la figure 1): l'un est maintenu dans un environnement contrôlé avec augmentation régulière des températures qui passent de $10^{\circ} \mathrm{C}$ en février à $22{ }^{\circ} \mathrm{C}$ en juin (TF); l'autre témoin est placé à l'extérieur et soumis aux thermo- et photo-périodes naturelles (TEX). Dans les conditions naturelles locales, les poissons rouges se reproduisent en juin et juillet.

A l'exception de ce dernier, tous les groupes sont soumis à une photopériode contrôlée (lampe fluorescente dont l'intensité est de 1000 lux à la surface de l'eau): en janvier, la durée d'éclairement est de $8 \mathrm{~h}$ par jour et est augmentée de $45 \mathrm{~min}$ tous les 15 jours pour atteindre $16 \mathrm{~h}$ en juillet. Chaque traitement a été répété trois fois, c'est-à-dire que pour chacun d'entre eux l'expérimentation a porté sur trois bassins de 10 animaux.

Le taux d'oxygène est maintenu au dessus de $6 \mathrm{ppm}$ par injection d'air comprimé dans les bassins. Les animaux sont nourris jusqu'à satiété trois fois par jour pour les groupes à 30 et $25^{\circ} \mathrm{C}$ et deux fois pour les autres avec des granulés pour truites 'Trouw.'

Les prélèvements de sang $(200 \mu \mathrm{l})$ sont pratiqués par ponction cardiaque à l'aide d'une seringue héparinée, 


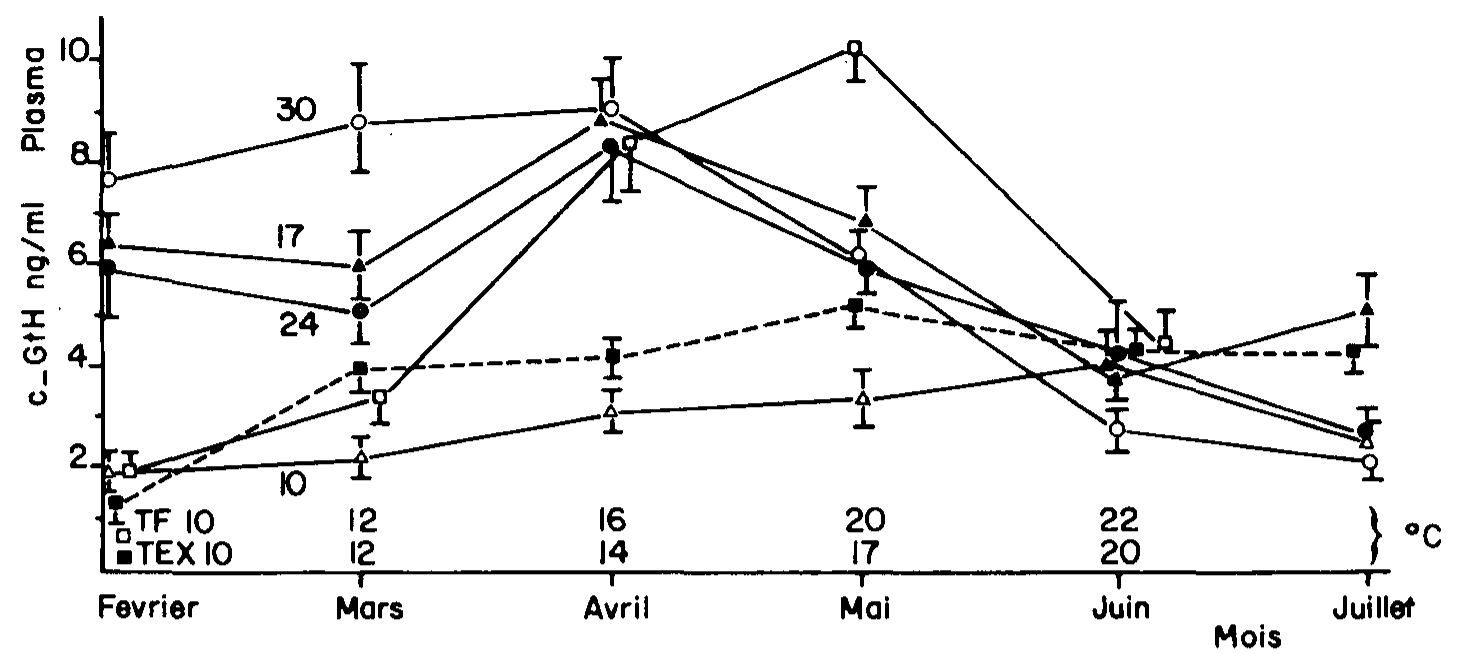

FIG. 1. Evolution de la teneur en $\mathrm{c}-\mathrm{GtH}$ plasmatique immunoréactive du poisson rouge entre février et juillet pour différentes températures d'élevage. TEX, témoin extérieur; TF, témoin maintenu en environnement contrôlé (cf. texte).

pendant 6 mois à raison d'une fois par mois (entre 9 et $12 \mathrm{~h}$ le matin). Après centrifugation, le plasma est stocké à $-20^{\circ} \mathrm{C}$ avant dosage radioimmunologique de la gonadotropine c-GtH (Breton et al. 1971). L'état de la gamétogenèse a été apprécié en avril (le 9) et juin (le 21) après sacrifice de 20 animaux par établissement du rapport gonadosomatique (RGS) et examen des testicules sur préparations histologiques. Sur des animaux, la charge hypophysaire en c-GtH a aussi été mesurée. Les comparaisons entre traitements ont été faites par analyse de variance. Les valeurs des graphiques sont accompagnées de l'erreur standard.

\section{Résultats}

Aux températures égales ou supérieures à $17^{\circ} \mathrm{C}$, les niveaux plasmatiques gonadotropes sont, dès le début de l'expérience, significativement plus élevés $(P<0.005)$ qu'à $10^{\circ} \mathrm{C}$ et atteignent des valeurs comprises entre 5 et 10 $\mathrm{ng} / \mathrm{ml}$. Cette situation se maintient en mars et avril, puis en juin et juillet les teneurs en c-GtH plasmatiques diminuent régulièrement jusqu'à $2-3 \mathrm{ng} / \mathrm{ml}$. A $10^{\circ} \mathrm{C}$ les teneurs passent progressivement de 2 à $4 \mathrm{ng} / \mathrm{ml}$ entre février et juin. Dans les groupes témoins, lorsque la température augmente lentement et reste au dessous de $20^{\circ} \mathrm{C}$ jusqu'en juin (groupe TEX) le niveau de c-GtH ne dépasse pas $5 \mathrm{ng} / \mathrm{ml}$. Au contraire, lorsque l'augmentation est plus rapide (groupe TF) on assiste à une forte augmentation de la sécrétion de gonadostimuline dès le mois d'avril lorsque la température atteint $16^{\circ} \mathrm{C}$. Les teneurs hypophysaires en c-GtH sont significativement plus élevées en juin qu'en avril $(P<0.05)$ pour les témoins extérieurs (TEX) et le groupe maintenu en perrmanence à $10^{\circ} \mathrm{C}$. Pour les autres températures expérimentales $\left(17,24\right.$ et $\left.30^{\circ} \mathrm{C}\right)$ les diffé- rences ne sont pas significatives entre avril et juin (fig. 2).

En ce qui concerne la gamétogenèse, le RGS des mâles est maximum en avril dans le groupe TEX et à $24^{\circ} \mathrm{C}$, mais les différences entre TEX, 10,17 et $24^{\circ} \mathrm{C}$ ne sont pas significatives; seul le RGS des individus à $30^{\circ} \mathrm{C}$ est significativement diminué par rapport aux précédents $(P<0.005)$ (fig. 3). De même, seuls les animaux maintenus à $30^{\circ} \mathrm{C}$ n'atteignent pas la maturité sexuelle. En juin, les RGS des groupes TEX et $10^{\circ} \mathrm{C}$ sont comparables et sont significativement plus élevés

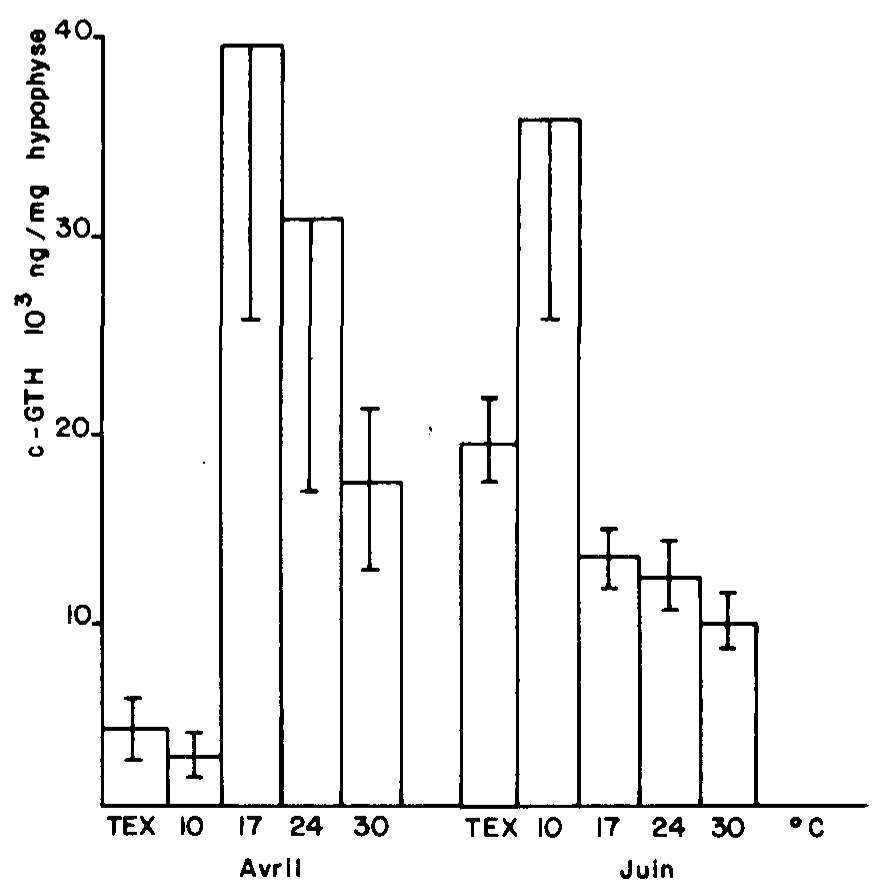

FIG. 2. Teneur hypophysaire en c-GtH chez le poisson rouge élevé depuis février dans des conditions thermiques différentes. 


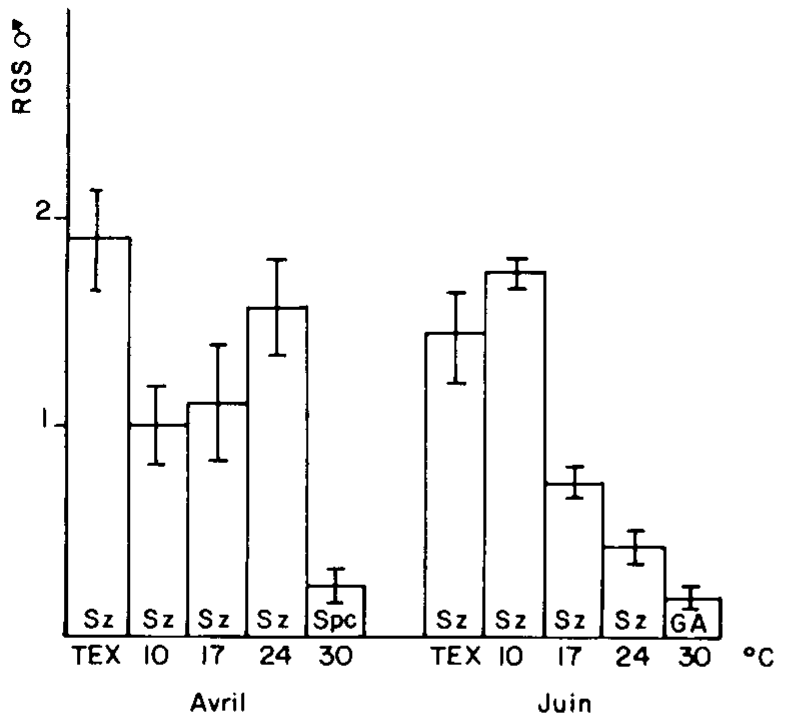

FIG. 3. Rapport gonadosomatique (RGS) de poissons rouges mâles. A la base des histogrammes figure le stade le plus avancé de la spermatogenèse identifié dans les testicules. Spc, spermatocytes; Sz, spermatozoïdes; GA, spermatogonies A.

$(P<0.005)$ que ceux des groupes 17,24 et $30{ }^{\circ} \mathrm{C}$. L'analyse histologique montre que du point de vue qualitatif la spermatogenèse est complète (apparition des spermatozoïdes dans tous les groupes sauf à $30^{\circ} \mathrm{C}$ ); à cette température le développement de la spermatogenèse est manifestement bloqué: en avril elle ne dépasse pas le stade spermatocyte qui n'est représenté que par quelques cystes. En juin, seules subsistent des spermatogonies A non organisées en cyste dans un testicule régressé où la structure lobulaire n'est plus visible.

\section{Discussion}

Les températures comprises entre 17 et $30^{\circ} \mathrm{C}$ stimulent au moins temporairement la sécrétion de la gonadotropine hypophysaire chez le poisson rouge; les niveaux gonadotropes circulants augmentent immédiatement après passage des animaux aux températures de 17,24 et $30^{\circ} \mathrm{C}$ et s'élèvent progressivement avec la température dans les témoins TF. Au contraire, l'augmentation de la c-GtH est faible dans le groupe maintenu en permanence à $10^{\circ} \mathrm{C}$. Ces effets favorables des températures élevées sur la sécrétion gonadotrope hypophysaire ne sont pas sans rappeler les réponses hypophysaires plus élevées obtenues en période estivale après injection de gonadotrope - releasing hormone (Gn-RH) chez la carpe (Weil et al. 1975). Les charges hypophysaires apparaissent en avril plus élevées à 17,24 et $30^{\circ} \mathrm{C}$ que dans les autres groupes élevés à une température plus basse, laissant supposer une synthèse accrue de c-GtH aux températures élevées. Cependant, il faut noter qu'en juin la charge est maximale à $10^{\circ} \mathrm{C}$, alors qu'une vidange hypophysaire s'est vraisemblablement produite dans les autres groupes. Les effets de la température se manifestent aussi au niveau des gonades et peuvent par là intervenir indirectement au niveau hypothalamohypophysaire; par exemple, à $30^{\circ} \mathrm{C}$ où la spermatogenèse est inhibée et n'aboutit jamais à la formation de spermatozoïdes bien que les gonadotropines circulantes soient élevées, on pourrait se trouver en présence d'une castration physiologique supprimant un rétro-contrôle des gonades sur les centres supérieurs comme cela a déjà été montré chez les poissons (Billard et al. 1976). Dans une large gamme de température comprise entre 10 et $24^{\circ} \mathrm{C}$, la spermatogenèse peut se dérouler normalement au moins du point de vue qualitatif, mais il semble qu'à 17 et $24^{\circ} \mathrm{C}$, il y ait une certaine accélération puisqu'à ces températures le RGS est maximum en avril et la spermatogenèse achevée en juin. A $10^{\circ} \mathrm{C}$, au contraire, le RGS est plus élevé en juin qu'en avril. Aux températures de 17 et $24^{\circ} \mathrm{C}$, il ne semble pas y avoir de poussées spermatogénétiques successives conduisant à une reproduction pratiquement continue sur une longue période comme chez la tanche (Breton et al. 1975). Les valeurs relativement faibles des RGS (les indices les plus élevés ne dépassent pas $2 \%$ ) pourraient être dues aux conditions d'élevage (confinement pendant une période prolongée, qualité de la nourriture, manipulations fréquentes). Un effet de la photopériode n'est pas à exclure; l'augmentation significative du niveau de gonadotropine plasmatique dans le groupe maintenu à $10^{\circ} \mathrm{C}$ laisse en effet la porte ouverte à une telle interprétation. Il faut cependant noter que la photopériode expérimentale imposée ne reproduit pas exactement la photopériode naturelle. La discussion ne pourra êtra poussée plus loin qu'après "une expérimentation complémentaire.

\section{Remerciements}

Ce travail a été réalisé dans le cadre de contrats C.E.E. No. 045.74.I ENV et E.D.F. No. 8375F $41 \mathrm{D} 97$.

BILLARD, R. 1968. Influence de la température sur la durée et l'efficacité de la spermatogenèse du Guppy Poecilia reticulata. C.R. Acad. Sci. 266: 2287-2290.

Billard, R., M. Richard et B. Breton. 1976. Stimula- 
tion de la sécrétion gonadotrope hypophysaire après castration chez la Truite Arc-en-ciel; variation de la réponse au cours du cycle reproducteur. C.R. Acad. Sci. Sér. D, 283: 171-174.

Breton, B., R. Billard et C. Weil. 1976. Facteurs du milieu et déterminisme endocrinien de la gamétogenèse chez les poissons cyprinidés et salmonidés. 2ème Coṇgrès Ichthyologistes Européen, Paris.

Breton, B., B. Jalabert, A. Fostier et R. Billard. 1975. Etude sur le cycle reproducteur de la Truite Arcen-ciel et de la Tanche. Effet de variations expérimentales de la température. J. Physiol. (Paris), 70: 561-564.

Breton, B., G. KanN, E. Burzawa-Gerard et R. Bil-
LARD. 1971. Dosage radioimmunologique d'une hormone gonadotrope de Carpe Cyprinus carpio L. C.R. Acad. Sci. 272: 1515-1517.

De Vlaming; V. L. 1974. Dans Environmental and endocrine control of teleost reproduction in control of sex in fishes. Rédigé par C. B. Schreck. VPI, Blacksburg, Virginia. pp. 13-83.

Weil, C., B. Breton et P. Reinaud. 1975. Etude de la réponse hypophysaire à l'administration de $\mathrm{Gn}-\mathrm{RH}$ exogène au cours du cycle reproducteur annuel chez la Carpe Cyprinus carpio L. C.R. Acad. Sci. 280: 2469-2472. 